\title{
Widespread Mechanic's Hands in Antisynthetase Syndrome With Anti-OJ Antibody
}

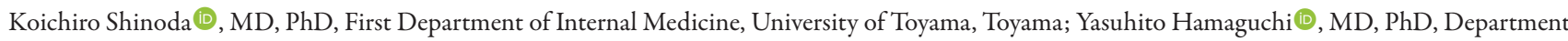
of Dermatology, Faculty of Medicine, Institute of Medical, Pharmaceutical and Health Sciences, Kanazawa University, Kanazawa; Kazuyuki Tobe (i), $\mathrm{MD}, \mathrm{PhD}$, First Department of Internal Medicine, University of Toyama, Toyama, Japan. Address correspondence to Dr. K. Shinoda, First Department of Internal Medicine, University of Toyama, 2630 Sugitani, Toyama 930-0194, Japan. Email: koichiro@med.u-toyama.ac.jp. The authors declare no conflicts of interest with regard to this work. The Ethics Committee of Toyama University Hospital approved this case report for submission (ethics approval number: E2020002). The patient gave written informed consent to publish the material.

Mechanic's hand is a representative manifestation of antisynthetase syndrome (AS) but is observed in other forms of dermatomyositis ${ }^{1}$. This is a nonpruritic, hyperkeratotic, and scaly eruption on the ulnar side of the thumb and radial side of other fingers; occasionally the palms, fingertips, and feet are involved. However, a widespread type affecting the whole palm and fingers is extremely rare in anti-OJ antibody.

A 66-year-old farmer was admitted with fever, cough, and eczema-like eruptions on the hands (Figure 1). Six months before admission, he received a course of methylprednisolone pulse therapy for interstitial lung disease, and his symptoms improved. A skin rash developed on both hands during the period of prednisolone (PSL) reduction to $10 \mathrm{mg} /$ day. When he was admitted, scaly hyperkeratosis was noted on each finger and on the lateral side and palm surface of both hands (Figure 1A). Skin biopsy revealed irregular acanthosis accompanied by hyperkeratosis and mild spongiosis (Figure 1C). Liquefaction degeneration and mucin deposition were not observed. Laboratory findings showed elevated muscle enzyme levels. Anti-OJ antibodies were identified by RNA and protein immunoprecipitation assays from the patient's serum ${ }^{2}$. Computed tomography showed a reticular shadow in the lower lung fields, consistent with AS. His symptoms improved with a course of methylprednisolone pulse therapy and $30 \mathrm{mg} /$ day of PSL. (Figure 1B). We considered his hand lesions as mechanic's hands. The skin biopsy findings were mostly compatible with those reported in a previous report ${ }^{3}$. In addition, his mechanic's hands was associated with disease activity.

\section{REFERENCES}

1. Targoff IN. Autoantibodies in polymyositis. Rheum Dis Clin North Am 1992;18:455-82.

2. Hamaguchi Y, Kuwana M, Takehara K. Comparison of anti-OJ antibody detection assays between an immunoprecipitation assay and line blot assay. Mod Rheumatol 2017;27:551-2.

3. Okiyama N, Yamaguchi Y, Kodera M, Hamaguchi Y, Yokozeki $\mathrm{H}$, Ishiguro $\mathrm{N}$, et al. Distinct histopathologic patterns of finger eruptions in dermatomyositis based on myositis-specific autoantibody profiles. JAMA Dermatol 2019;155:1080-2.
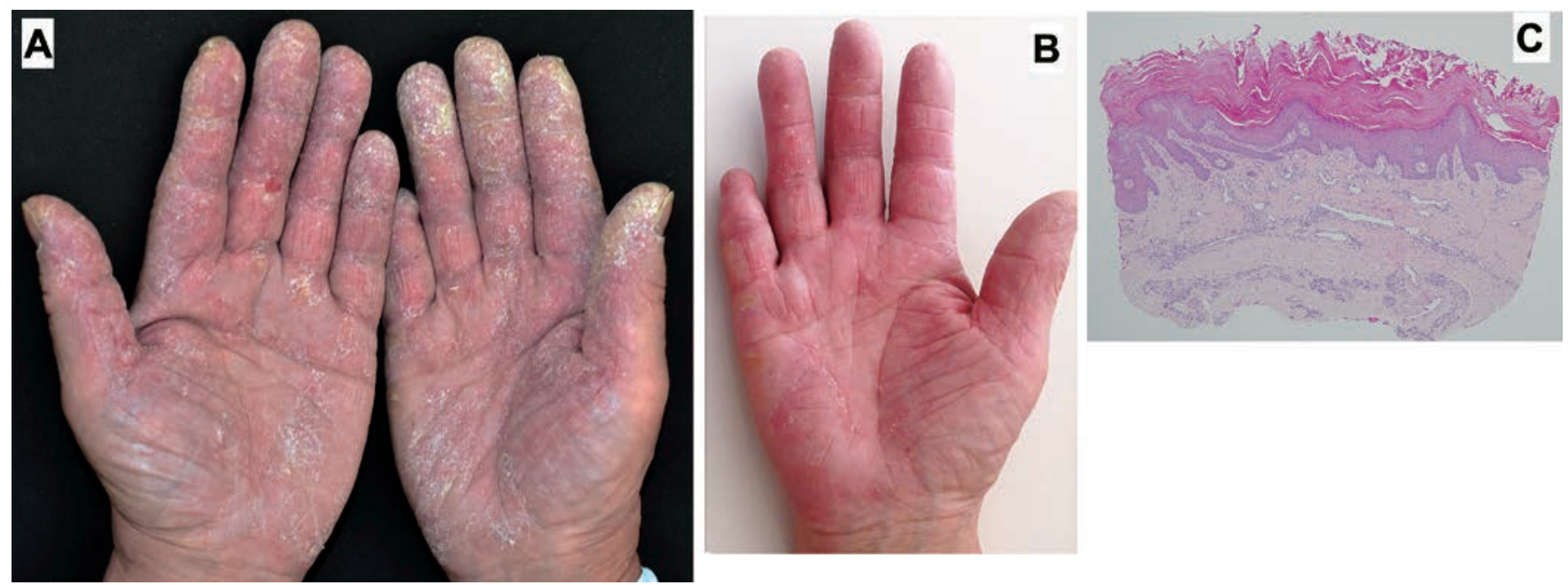

Figure 1. (A) Skin examination on admission demonstrates scaly hyperkeratosis on each finger as well as the lateral side and palm surface of both hands, called "widespread mechanic's hand." (B) Skin examination 2 weeks after corticosteroid therapy demonstrates drastic improvement of the mechanic's hand. (C) Skin biopsy revealed irregular acanthosis accompanied by hyperkeratosis and mild spongiosis. 